\title{
The Effects of WISPI Polymorphisms on the Prognosis of Lung Cancer Patients with Platinum-Based Chemotherapy
}

\author{
Jia $\mathrm{He}^{\prime}$ \\ Zhan Wang $^{2}$ \\ Ying Wang ${ }^{3}$ \\ Ting Zou ${ }^{4}$ \\ Xiang-Ping $\mathrm{Li}^{1}$ \\ Lei Cao' \\ Juan Chen' \\ 'Department of Pharmacy, Xinagya \\ Hospital, Central South University, \\ Changsha, 410008, People's Republic of \\ China; ${ }^{2}$ Department of Medical \\ Oncology, Lung Cancer and \\ Gastrointestinal Unit, Hunan Cancer \\ Hospital, Affiliated Cancer Hospital of \\ Xiangya School of Medicine, Changsha, \\ 410013, People's Republic of China; \\ ${ }^{3}$ Hunan Clinical Research Center in \\ Gynecologic Cancer, Hunan Cancer \\ Hospital, Affiliated Cancer Hospital of \\ Xiangya School of Medicine, Changsha, \\ 4I00I3, People's Republic of China; \\ ${ }^{4}$ National Institution of Drug Clinical \\ Trial, Xiangya Hospital, Central South \\ University, Changsha, 410008, People's \\ Republic of China
}

Purpose: To investigate the relationships between Wnt1 inducible signaling pathway protein 1 (WISP1) polymorphisms and the prognosis of platinum-based chemotherapy in lung cancer patients.

Patients and Methods: A total of 363 lung cancer patients were recruited in this study. All of them received at least two cycles of platinum-based chemotherapy. We used unconditional logistic regression analysis to assess the associations of 39 single nucleotide polymorphisms in WISP1 gene with platinum-based chemotherapy prognosis.

Results: The results indicated that patients carried rs2929973 GT or GG genotypes had increased risk of disease progression ( $\mathrm{HR}=0.712,95 \% \mathrm{CI}=0.553-0.916, P=0.015$ ). Patients with rs2977551 TT genotype had a significantly decreased risk of progression-free survival than patients carrying CT or CC genotype $(\mathrm{HR}=0.723,95 \% \mathrm{CI}=0.561-0.932, P=0.032)$ and overall survival $(\mathrm{HR}=0.725,95 \% \mathrm{CI}=0.552-0.913, P=0.045)$. For rs2977549, patients carrying TT genotype had a significantly longer progression-free survival than patients with $\mathrm{CC}$ or $\mathrm{CT}$ genotypes $(\mathrm{HR}=0.708,95 \% \mathrm{CI}=0.550-0.912, P=0.017)$. Among of them, rs16904853, rs10956697, rs2929965, rs2929973, rs7828685, rs2977551 and rs2977549 were related to progression-free survival, and rs10956697 and rs2977551 were related to overall survival in subgroup analyses, respectively.

Conclusion: WISP1 rs2929973, rs2977551 and rs2977549 may be contributed to a potential candidate biomarker for prediction of platinum-based chemotherapy prognosis in lung cancer patients.

Keywords: lung cancer, platinum-based chemotherapy, prognosis, genetic polymorphism, WISP1

\section{Introduction}

Lung cancer is the most common incidence and the leading cause of cancer related deaths worldwide. ${ }^{1}$ Two major types of lung cancer are classified as small cell lung cancer (SCLC) and non-small cell lung cancer and (NSCLC). ${ }^{2}$ NSCLC patients, approximately $85 \%$ of the total lung cancer cases, are often diagnosed in advanced stage and metastatic. ${ }^{3,4}$ At present, the main therapies of lung cancer are surgery, radiation therapy, immunotherapy and chemotherapy. ${ }^{5}$ Immunomodulatory therapies have been approved as second-line agents for patients with advanced lung cancer as well as first-line therapy for patients with high level $(>50 \%)$ of PD-L1 expression and absence of sensitizing EGFR mutations or ALK rearrangements. ${ }^{6}$ However, lung cancer patients with advanced NSCLC who do not fit an approved
Correspondence: Juan Chen Department of Pharmacy, Xiangya Hospital, Central South University, Changsha, 410008, People's Republic of China

Tel/Fax +86-73I-8975349l

Email cj1028@csu.edu.cn 
molecular targeted therapy, the standard first-line treatment remains platinum-based doublet therapy with or without bevacizumab. ${ }^{5}$ The five-year overall survival rate for lung cancer is still approximately $18 \%$, the prognosis for the advanced-stage disease is even poorer, with a median overall survival of approximately one year. ${ }^{7,8}$

Wnt1 inducible signaling pathway protein 1 (WISP1), as a member of the CCN family of proteins, is known as CCN4. ${ }^{9}$ The human WISP1 gene is located on chromosome 8q24.1-q24.3. ${ }^{10}$ The CCN family of proteins possess six secreted extracellular matrix associated proteins. Each family member contains four structural domains: an insulin-like growth factor binding protein-like module (IGFBP), a von Willebrand factor type C repeat (VWC), a thrombospondin domain (TSP1), and a C-terminal cysteine-knot-containing (CT) domain. ${ }^{11}$ Wntl signaling pathways can modulate multiple processes that involve neuronal development, angiogenesis, immune cell modulation, tumorigenesis, and stem cell proliferation. ${ }^{12-15}$ Activation of WISP1 transcript and protein were detected in carcinomas in vivo, such as hepatocellular carcinoma, colon adenocarcinomas, lung carcinoma, and breast cancer. ${ }^{10,16-18}$ However, the role of WISP1 in clinical lung cancer with platinum-based chemotherapy still needs to be clarified.

Single Nucleotide Polymorphisms (SNPs) represent the most common type of variation in the human genome, a single base in the DNA differs from the usual base at that position. SNPs are the marker of choice in genetic analysis and also useful in locating genes association with diseases. ${ }^{19}$ While the reason of SNPs effects on gene expression, protein binding is not completely understood. $^{20}$ Previous study reported that WISP1 has been detected in scirrhous gastric carcinoma and invasive cholangiocarcinoma. ${ }^{21}$ Overexpression of WISP1 variants increases the growth rate of mouse embryonic fibroblasts rat kidney fibroblasts, hBMSCs and human esophageal cancer cells. ${ }^{21,22}$ In lung cancer cells overexpression WISP1 leads to an inhibition in vitro cell invasion and motility, as well as lung metastasis. ${ }^{17}$ The roles of WISP1 were marked varieties in difference type of cancers. For example, high expression levels of WISP1 were correlated with poor prognosis in breast, ${ }^{18}$ rectal, ${ }^{23}$ esophageal cancer ${ }^{24}$ and colon cancer, ${ }^{25}$ but WISP1 expressed lower levels in melanoma with poor prognosis. ${ }^{26}$ Despite the role of WISP1 affects lung cancer progression is not clear.

In the present study, we evaluated whether genotypes of WISP1 in lung cancer patients could be involved in prognosis of lung cancer patients with platinum-based chemotherapy.

\section{Methods and Materials}

\section{Study Populations}

The study subjects consist of 363 patients that are diagnosed lung cancer in the Affiliated Cancer Hospital or Xiangya Hospital of Central South University (Changsha, Hunan, China) during 2011 to 2013. Patients clinical and laboratory findings were collected from medical records. The recruitment criteria included the following: Eligible patients were histologically or cytologically proven lung cancer who had experienced disease progression. Patients with a history of previous malignant tumor or concomitant malignancies, metastases were excluded from this cohort. All patients were treated with platinum-based chemotherapy at least 2 cycles and were not given radiotherapy and/ or biological therapy before and during chemotherapy. All patients were signed informed consents were obtained from all study participants before chemotherapy. The clinical and laboratory data including age, sex, smoking history, TNM stage, histological type and stage, chemotherapy regimens were collected from medical records.

The study was in accordance with the ethical standards of the institutional and/or national research committee and with the 1964 Helsinki declaration, and the study protocol was approved by the Ethics Committee of Xiangya School of Medicine, Central South University with a registration number of CTXY-110008-2.

\section{SNP Selection, DNA Extraction and Genotyping}

According to the data from the HapMap, or previous studies, we selected 39 SNPs (Table S1) of the WISP1 gene which described previously. ${ }^{27,28}$ In brief, the following criteria: (1) A total of them with minor allele frequency (MAF) $\geq 0.05$ in the Chinese population were selected. (2) The tag SNP selection was performed using the Tagger program implemented in Haploview version 4.2. (3) SNPs located in the promoter region, exon region, and the 3' untranslated region (UTR). Genomic DNA was extracted from the whole blood by using the FlexiGene DNA Kit (Qiagen, Hilden, Germany) according to the manufacturer's instructions. The DNA sample was stored at $-20{ }^{\circ} \mathrm{C}$ before usage. WISP1 polymorphism was 
genotyped using the Sequenom Mass ARRAY System (Sequenom, San Diego, CA, USA).

\section{Statistical Analysis}

The statistical analysis was conducted by the SPSS version 25.0 (SPSS Inc., Chicago, IL, USA) and GraphPad Prism (version 8, GraphPad Software Inc., San Diego, CA). We used Cox proportional hazards regression model to analysis each genetic variant and OS/PFS, measured as HRs with their corresponding $95 \% \mathrm{CIs}$. The covariates used for adjusted HR for PFS included age, gender, smoking status, histology, and clinical stage between the OS and PFS. Kaplan-Meier test was used to assess each genetic variant on the cumulative probability of PFS and OS. All association analyses were evaluated by three models including additive, dominant, and recessive. All P-values presented were two-sided, and a level of $P<0.05$ was considered statistically significant.

\section{Results}

\section{Characteristics and Survival Status of the Lung Cancer Patients}

In order to investigate the association of WISP1 Polymorphisms and clinical results of platinum-based chemotherapy, the present study consisted of 363 patients diagnosed with lung cancer, who had DNA samples, complete data on demographic, clinical characteristics, progression free survival (PFS) and overall survival (OS). All patients who received at least 2 cycles of platinumbased chemotherapy were enrolled to investigate the association of WISP1 polymorphisms with chemotherapy. As shown in Table 1, 289 were males and 74 were females, with the median age of cohort was 56 (a range of 21 to 77 years old). The patients who ever or current smokers accounted for $63.6 \%$, and the ones who were never smokers accounted for $36.4 \%$. For the patient group, 244 (67.2\%) patients with presented advanced NSCLC and $119(32.8 \%)$ patients with SCLC, and most of which were with stage III/IV (65.8\%).

\section{Association of WISPI Polymorphisms with Platinum-based Chemotherapy Prognosis in the Lung Cancer Patients}

To investigate the association between WISP1 Polymorphisms and the prognosis of lung cancer patients received platinum-based chemotherapy, we selected 39 SNPs and those SNPs under investigation are shown in Table 2. After adjustments for age, sex, smoking status, histological type and TNM stage 7 SNPs (rs16904853, rs10956697, rs2929965, rs2929973, rs7828685, rs2977551, rs2977549) consistently showed a significant association with PFS and/or OS in lung cancer patients using Log rank test and multivariate Cox regression analysis (Table 2). Unconditional logistic regression analysis with adjustments revealed that WISP1 rs16904853 ( $P$ for log-rank=0.010), rs10956697 $(P$ for $\log$-rank=0.014), rs2929965 $(P$ for log-rank=0.036), rs7828685 ( $P$ for logrank $=0.025$ ) were significantly associated with lung cancer PFS in the additive model. WISP1 rs10956697 ( $P$ for logrank=0.004), $\quad r 2929973 \quad(P$ for $\log$-rank=0.015), rs7828685 ( $P$ for log-rank=0.027), rs2977551 ( $P$ for logrank=0.032), rs2977549 ( $P$ for log-rank=0.017) were significantly related to lung cancer PFS in the dominant model. rs16904853 ( $P$ for log-rank=0.003), rs2929965 $(P$ for log-rank=0.013), rs7828685 $(P$ for log-rank $=0.023$ ) were significant association with PFS in the recessive model (Figure 1). For OS analysis, our results demonstrated that lung cancer patients with rs 10956697 were significantly related to better $\mathrm{OS} \quad(\mathrm{HR}=0.728,95 \%$ $\mathrm{CI}=0.557-0.953, \quad P=0.021)$ in the dominant model. Additionally, patients carrying TT genotype in rs2977551 were significantly related to better $\mathrm{OS}(\mathrm{HR}=0.725,95 \%$ $\mathrm{CI}=0.552-0.913, P=0.020$ ) (Table 2) (Figure 2).

\section{Association Between SNPs and Lung Cancer Progression}

Next, we evaluated the relationship between the single nucleotide polymorphisms were related to tumor progression with patients receiving platinum-based chemotherapy. As described in Table 3, the rs2929973, rs2977551 and rs2977549 in WISP1 gene still significantly contributed to $\mathrm{T}$ stage of lung cancer patients. Both in the univariate Cox regression analysis and after adjusted by age, gender, smoking status, histological type and stage, we found that patients carrying GT or GG genotype in rs2929973 were a lower hazard to early $\mathrm{T}$ stage (T1 or T2) when compared with TT genotype $(\mathrm{OR}=0.486,95 \% \mathrm{CI}=0.271-$ $0.871, P=0.015$ ). Patients who carried the rs $2977551 \mathrm{CT}$ or CC genotype had a more significantly association with early T stage than patients with TT genotype $(\mathrm{OR}=0.428$, 95\% CI $=0.238-0.771, P=0.005$ ). For $r 2977549$ compared with CT or CC genotype carries, individuals with TT genotype tended to be association with early $\mathrm{T}$ stage $(\mathrm{OR}=0.438,95 \% \mathrm{CI}=0.246-0.781, P=0.005)$. 
Table I Association Between Clinical Characteristics and PFS and OS of Patients with Lung Cancer

\begin{tabular}{|c|c|c|c|c|c|c|c|c|c|c|}
\hline \multirow[t]{2}{*}{ Variables } & \multicolumn{5}{|c|}{ Overall Survival (OS) } & \multicolumn{5}{|c|}{ Progression-Free Survival (PFS) } \\
\hline & MST (Year) & Death/Total & $\mathbf{P I}$ & HR (95\% Cl) & $\mathbf{P 2}$ & MST (Year) & Progression/Total & PI & HR (95\% Cl) & $\mathbf{P 2}$ \\
\hline $\begin{array}{l}\text { Age (year) } \\
\quad<60 \\
\geq 60\end{array}$ & $\begin{array}{l}4.28 \\
4.33\end{array}$ & $\begin{array}{l}159 / 164 \\
94 / 97\end{array}$ & 0.133 & $\begin{array}{l}\text { Ref. } \\
0.82(0.63-1.06)\end{array}$ & 0.134 & $\begin{array}{l}4.32 \\
4.07\end{array}$ & $\begin{array}{l}178 / 242 \\
102 / 130\end{array}$ & 0.611 & $\begin{array}{l}\text { Ref. } \\
1.06(0.83-1.36)\end{array}$ & 0.611 \\
\hline $\begin{array}{l}\text { Gender } \\
\text { Female } \\
\text { Male }\end{array}$ & $\begin{array}{l}4.62 \\
4.10\end{array}$ & $\begin{array}{l}49 / 51 \\
204 / 210\end{array}$ & 0.218 & $\begin{array}{l}\text { Ref. } \\
0.82(0.60-1.12)\end{array}$ & 0.219 & $\begin{array}{l}4.98 \\
3.89\end{array}$ & $\begin{array}{l}44 / 76 \\
135 / 296\end{array}$ & 0.287 & $\begin{array}{l}\text { Ref. } \\
0.85(0.64-1.14)\end{array}$ & 0.288 \\
\hline $\begin{array}{l}\text { Smoking status } \\
\text { Never smoker } \\
\text { Ever smoker }\end{array}$ & $\begin{array}{l}4.62 \\
3.72\end{array}$ & $\begin{array}{l}91 / 93 \\
161 / 167\end{array}$ & 0.393 & $\begin{array}{l}\text { Ref. } \\
0.89(0.69-1.16)\end{array}$ & 0.394 & $\begin{array}{l}4.83 \\
3.72\end{array}$ & $\begin{array}{l}101 / 137 \\
178 / 234\end{array}$ & 0.226 & $\begin{array}{l}\text { Ref. } \\
0.86(0.67-1.10)\end{array}$ & 0.227 \\
\hline $\begin{array}{l}\text { Histology } \\
\text { NSCLC } \\
\text { SCLC }\end{array}$ & $\begin{array}{l}4.36 \\
4.06\end{array}$ & $\begin{array}{l}137 / 179 \\
77 / 79\end{array}$ & 0.668 & $\begin{array}{l}\text { Ref. } \\
\text { I.06(0.8I-1.39) }\end{array}$ & 0.668 & $\begin{array}{l}3.92 \\
4.81\end{array}$ & $\begin{array}{l}193 / 250 \\
87 / 122\end{array}$ & 0.289 & $\begin{array}{l}\text { Ref. } \\
0.87(0.68-1.12)\end{array}$ & 0.289 \\
\hline $\begin{array}{l}\text { TNM stage } \\
\text { I/II } \\
\text { III/IV } \\
\text { LD } \\
\text { ED }\end{array}$ & $\begin{array}{l}3.94 \\
4.42 \\
3.50 \\
4.10\end{array}$ & $\begin{array}{l}6 / 6 \\
169 / 175 \\
33 / 34 \\
43 / 44\end{array}$ & $\begin{array}{l}0.764 \\
0.905\end{array}$ & $\begin{array}{l}\text { Ref. } \\
0.88(0.39-2.00) \\
\text { Ref. } \\
\text { I.03(0.65-1.63) }\end{array}$ & $\begin{array}{l}0.765 \\
0.905\end{array}$ & $\begin{array}{l}4.07 \\
3.89 \\
4.31 \\
4.89\end{array}$ & $\begin{array}{l}7 / 11 \\
148 / 239 \\
37 / 52 \\
49 / 67\end{array}$ & $\begin{array}{l}0.416 \\
0.862\end{array}$ & $\begin{array}{l}\text { Ref. } \\
\text { I.37(0.64-2.91) } \\
\text { Ref. } \\
\text { I.04(0.68-1.60) }\end{array}$ & $\begin{array}{l}0.418 \\
0.862\end{array}$ \\
\hline
\end{tabular}

Notes: PI, P-value for Log rank test; P2, P-value for univariate Cox hazards regression analysis; $\mathrm{P}<0.05$ are indicated in bold text.

Abbreviations: MST, median survival time (year); HR, hazard ratio; $\mathrm{Cl}$, confidence interval; Ref., reference; NSCLC, lung adenocarcinoma; SCLC, lung squamous cell carcinoma.

\section{Subgroup Analysis of Association Between WISPI Polymorphisms and Prognosis}

To better explore the WISP1 polymorphisms with OS and PFS of patients received chemotherapy, stratified analysis was performed based on the ages, gender, smoking status, histological type and TNM stage.

As shown in Table 4, the risk genotype group carries with rs2929973 GT or GG genotypes tended to have a significantly increased risk of disease progression and PFS in subgroups of patients with $\leq 60$ years old. For patients who had never smoked, carrying TT genotype had a significantly decreased risk of death $(\mathrm{HR}=0.617$, 95\% CI $=0.383-0.994, P=0.047$ ). In the presence of GT or GG genotype, patients with NSCLC were markedly increased than those with TT genotype ( $\mathrm{HR}=0.693,95 \%$ $\mathrm{CI}=0.501-0.959, P=0.027$ ). For patients who had TNM stage III/IV, carrying GT or GG genotype had a significantly increased risk of death (HR $=0.649,95 \%$ $\mathrm{CI}=0.470-0.896, P=0.009)$ and PFS $(\mathrm{HR}=1.377,95 \%$ $\mathrm{CI}=1.024-1.852, P=0.034)$. For $\mathrm{rs} 2977551$, we found that carriers of $\mathrm{CT}$ or $\mathrm{CC}$ genotype tended to have a significantly increased risk of PFS in subgroups of younger $(\leq 60$ years old $)(\mathrm{HR}=1.413,95 \% \mathrm{CI}=1.022$
1.954, $P=0.037)$, never smokers $(\mathrm{HR}=1.572,95 \%$ $\mathrm{CI}=1.007-2.455, P=0.047)$. For rs2977549, the risk genotype group carriers with $\mathrm{CT}$ or $\mathrm{CC}$ genotypes tended to have a significantly increased risk of disease progression in subgroups of patients with $\leq 60$ years old. Patients carrying TT genotype tend to a lower risk of PFS ( $\mathrm{HR}=1.480,95 \% \mathrm{CI}=1.071-2.047, P=0.018)$. Compared with CT or CC genotype tend to be sensitive to platinumbased chemotherapy, patients with NSCLC were markedly increased risk of death than those with TT genotype $(\mathrm{HR}=1.502,95 \% \mathrm{CI}=1.087-2.075, P=0.014)$ and $\mathrm{PFS}$ $(\mathrm{HR}=1.358,95 \% \mathrm{CI}=1.005-1.835, P=0.046)$. For patients who had TNM stage III/IV, rs2977549 carrying TT genotype had a significantly decreased risk of death $(\mathrm{HR}=1.601,95 \% \mathrm{CI}=1.157-2.216, P=0.005)$ and $\mathrm{PFS}$ $(\mathrm{HR}=1.419,95 \% \mathrm{CI}=1.053-1.913, P=0.022)$.

\section{Discussion}

Our present study systematically investigated the clinical significances of the WISP1 polymorphisms on the prognosis of lung cancer patients that underwent platinumbased chemotherapy. In the current study, we found that WISP1 rs2929973, rs2977551 and rs2977549 contribute to a significantly correlated with platinum-based prognosis. 


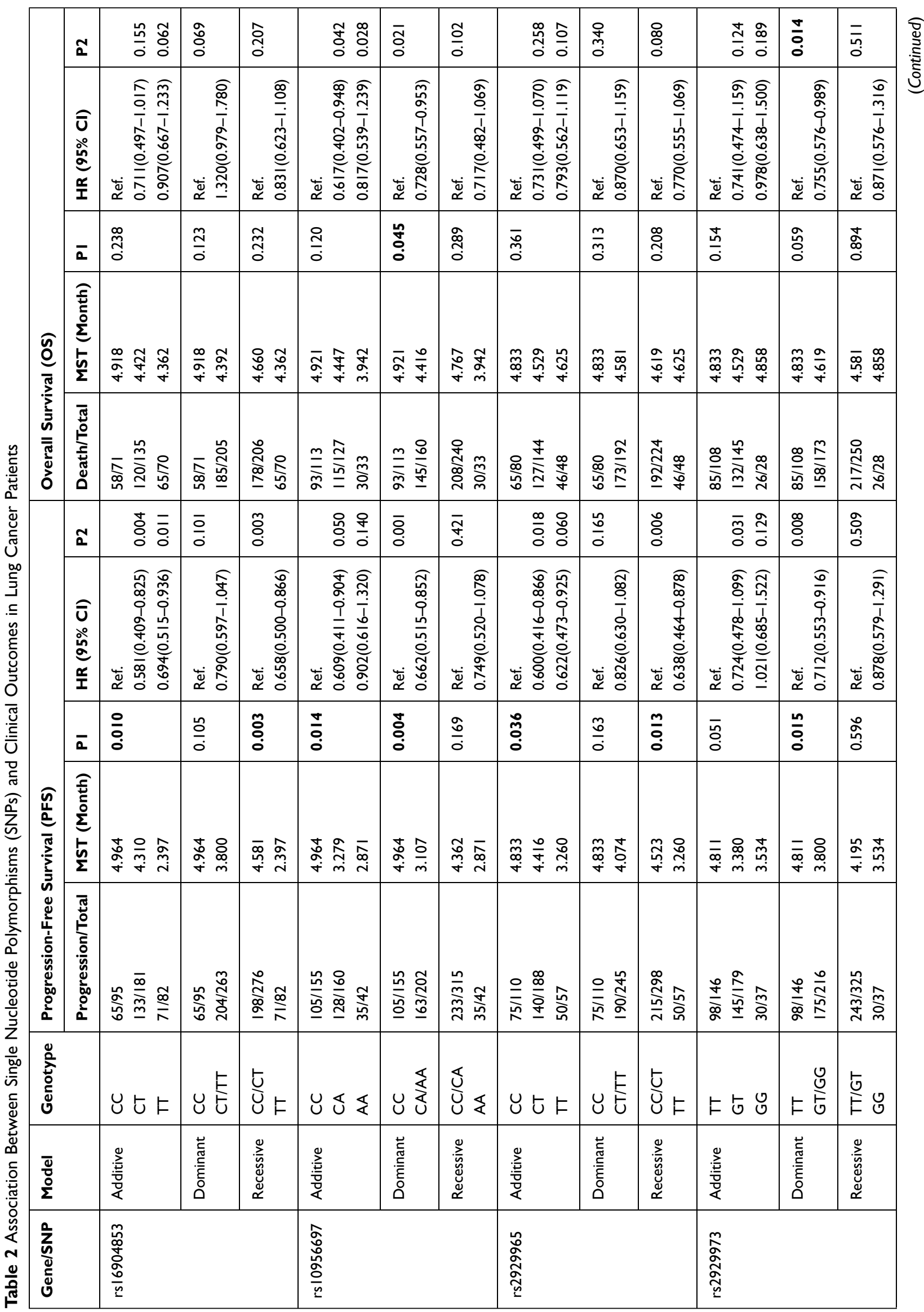




\begin{tabular}{|c|c|c|c|c|c|c|c|c|c|c|c|}
\hline \multirow{5}{*}{ 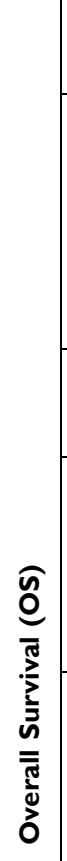 } & $\Sigma$ & $\begin{array}{l}0 \\
0 \\
0 \\
0\end{array}$ & ồ. & $\frac{0}{0}$ & 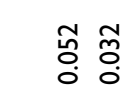 & : & $\frac{\infty}{\frac{\infty}{0}}$ & $\begin{array}{l}\text { ơ } \\
\text { o̊ } \\
0\end{array}$ & Õ & 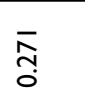 & | \\
\hline & 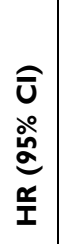 & 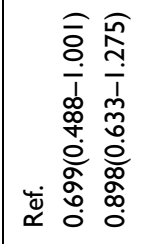 & 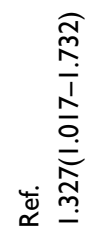 & 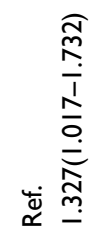 & 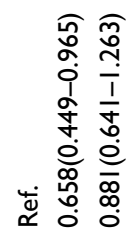 & 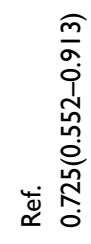 & 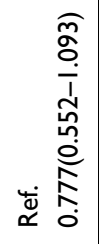 & 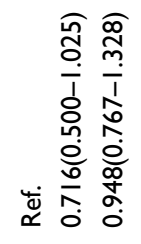 & 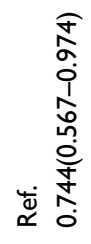 & 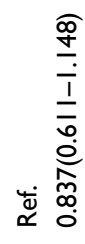 & 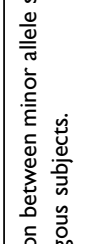 \\
\hline & $\bar{a}$ & $\frac{m}{0}$ & $\begin{array}{l}\text { @ } \\
: \\
0\end{array}$ & $\frac{\hat{E}}{0}$ & $\stackrel{ \pm}{\frac{ \pm}{0}}$ & 苂 & ণัণ & $\frac{\dot{2}}{0}$ & 资 & 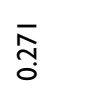 & \\
\hline & 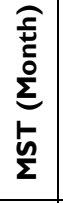 & 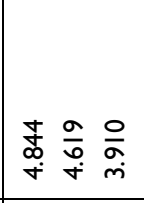 & 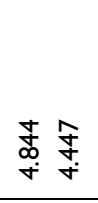 & 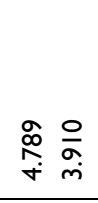 & 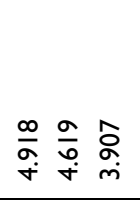 & 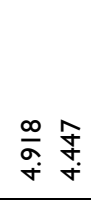 & 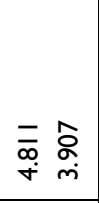 & 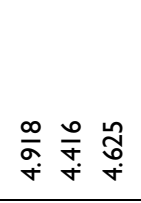 & \begin{tabular}{ll}
$\frac{\infty}{a}$ & \multirow{f}{+}{} \\
$\dot{+}$ & + \\
\end{tabular} & $\begin{array}{l}0 \\
\stackrel{0}{0} \\
\stackrel{0}{0} \\
\stackrel{+}{+} \\
\stackrel{+}{+}\end{array}$ & 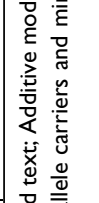 \\
\hline & 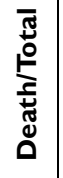 & 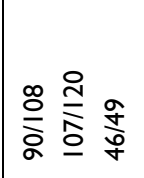 & 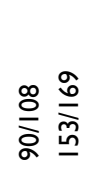 & 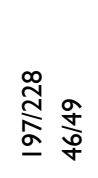 & 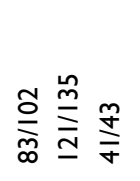 & 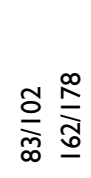 & 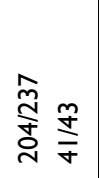 & 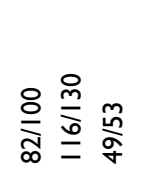 & 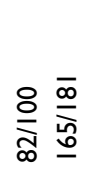 & 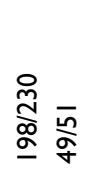 & $\frac{\widetilde{J}}{\bar{g}}$ \\
\hline \multirow{5}{*}{ 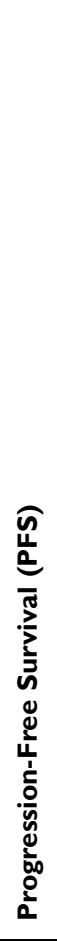 } & $\Sigma$ & 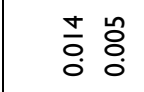 & $\frac{0}{0}$ & 志 & $\begin{array}{l}\hat{n} \\
\hat{0} \\
0 \\
0\end{array}$ & $\frac{0}{0}$ & $\frac{\tilde{0}}{0}$ & 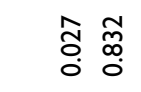 & 啇 & $\underset{\substack{n \\
0}}{0}$ & \\
\hline & 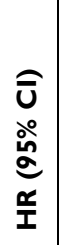 & 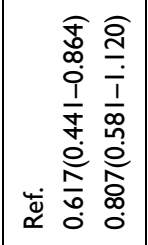 & 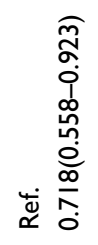 & 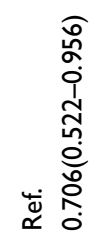 & 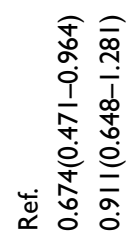 & 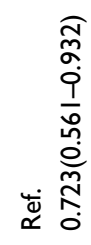 & 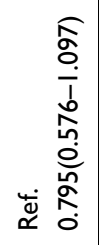 & 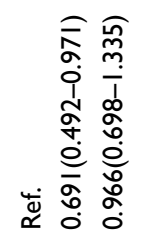 & 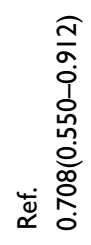 & 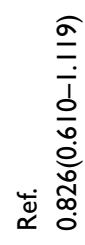 & 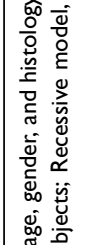 \\
\hline & $\bar{a}$ & 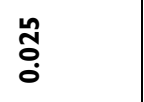 & $\hat{\Xi}$ & $\begin{array}{l}n \\
\vdots \\
0 \\
0\end{array}$ & $\stackrel{\circ}{\circ}$ & $\begin{array}{l}\tilde{o} \\
0 \\
0\end{array}$ & ثัণ & 垈 & no & $\frac{m}{\pi}$ & \\
\hline & 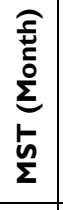 & 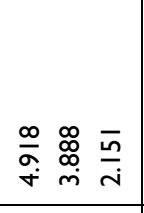 & 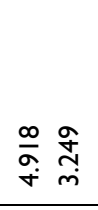 & $\underset{+}{+} \frac{\bar{n}}{i}$ & 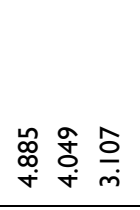 & 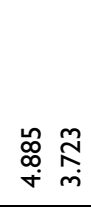 & 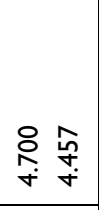 & 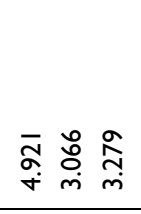 & 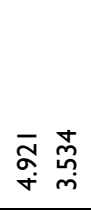 & 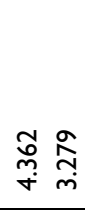 & 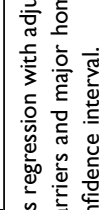 \\
\hline & 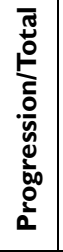 & 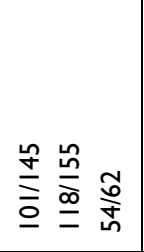 & 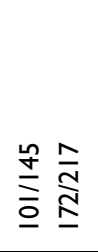 & $\begin{array}{l}\frac{8}{0} \\
\frac{\mathrm{m}}{\mathrm{N}} \\
\frac{\mathrm{o}}{\mathrm{N}}\end{array}$ & 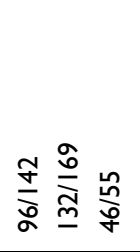 & 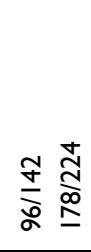 & 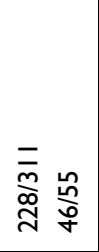 & 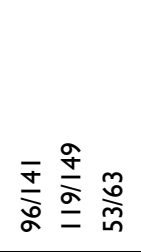 & 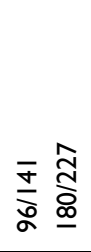 & 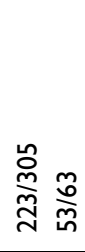 & : \\
\hline 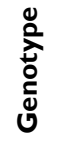 & & \&芯 & 焉哥 & 芯 & $\vDash \leftarrow U$ & $\vdash \stackrel{U}{\stackrel{U}{U}}$ & $\stackrel{E}{E} \cup$ & $\vDash ヒ U$ & $\vDash \stackrel{\stackrel{U}{U}}{U}$ & $\stackrel{E}{E}$ & \\
\hline $\begin{array}{l}\bar{d} \\
\frac{0}{0} \\
\Sigma\end{array}$ & & $\frac{\sum_{i}^{0}}{+\frac{7}{2}}$ & 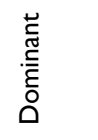 & 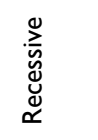 & $\frac{\stackrel{0}{2}}{\frac{i}{2}}$ & 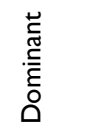 & 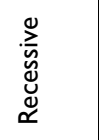 & $\frac{\stackrel{0}{Z}}{\frac{i}{+0}}$ & 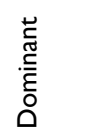 & 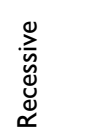 & \\
\hline $\begin{array}{l}\frac{0}{z} \\
\frac{1}{0} \\
\mathbb{E} \\
0 \\
0\end{array}$ & & 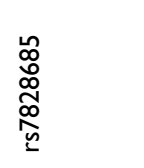 & & & 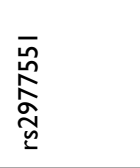 & & & 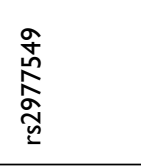 & & & \\
\hline
\end{tabular}


A Additive

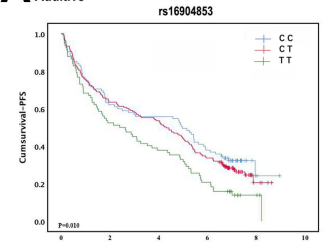

B Dominan

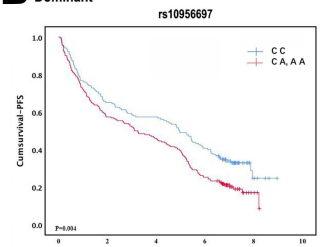

C Recessive ${ }^{\text {rimetyear) }}$

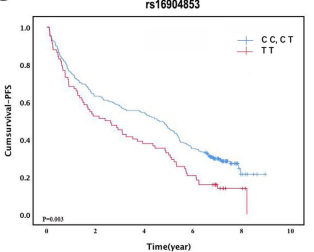

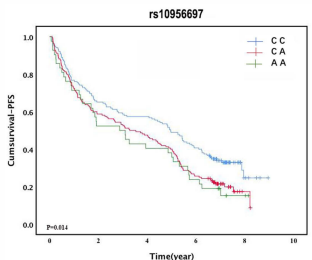

Timelyear)

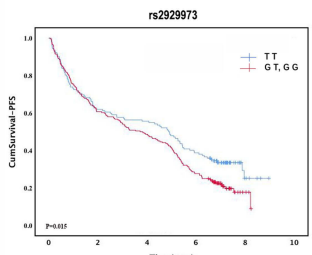

rs2929965

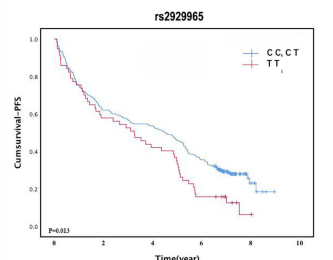

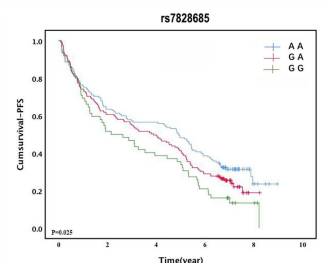

Time(year)

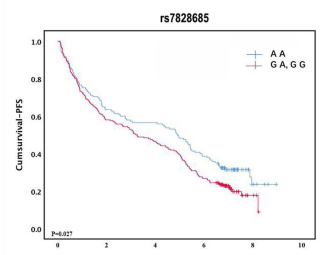

Timelyear)

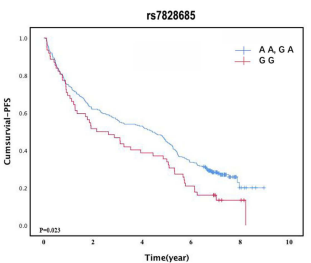

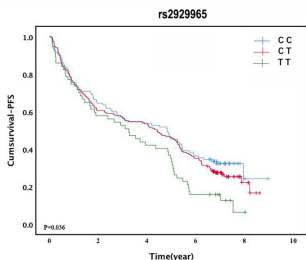
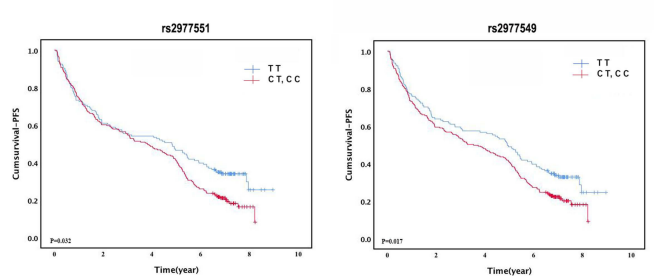

Figure I Kaplan-Meier plots of lung cancer patients with different genotypes of in WISPI gene. (A) PFS of lung cancer patients by rs I6904853, rs I0956697, rs7828685, rs2929965 genotypes using additive model. (B) PFS of lung cancer patients by rs 10956697, rs2929973, rs7828685, rs297755I, rs2977549 genotypes using dominant model. (C) PFS of lung cancer patients by rs 16904853, rs2929965, rs7828685 genotypes using recessive model.

A

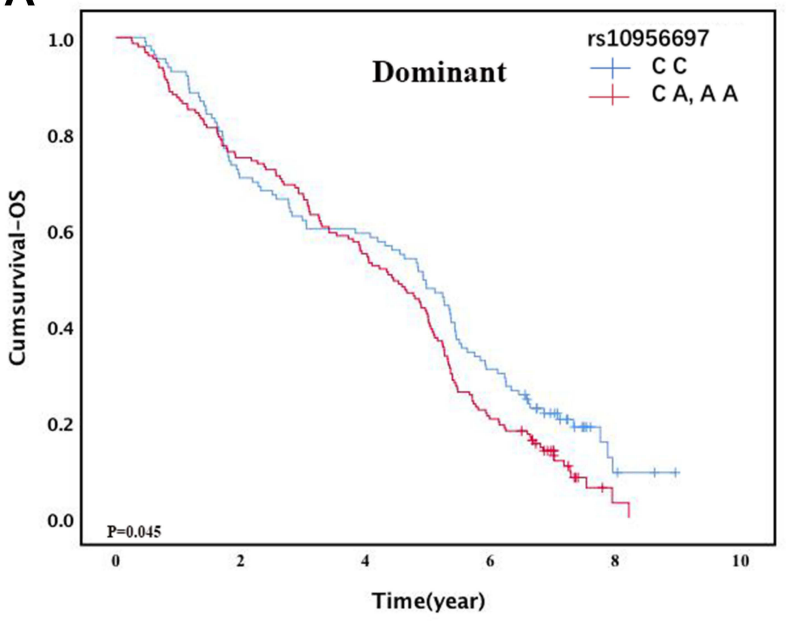

B

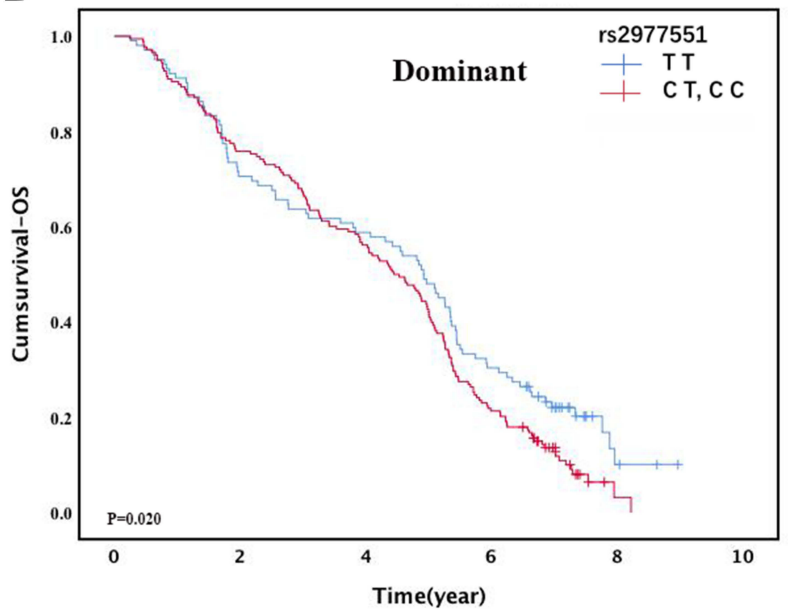

Figure 2 Kaplan-Meier plots of lung cancer patients with different genotypes of in WISPI gene. (A) OS of lung cancer patients by rs I0956697 using dominant model. (B) OS of lung cancer patients by rs297755I using dominant model.

In conclusion, our investigation provided the useful basics to make more precise evaluation of the chemotherapy efficacy and then further design personalized therapy.

The differential expression status of WISP1 between the tumor tissue and normal healthy tissue. ${ }^{9}$ High levels of WISP1 correlated with survival in prostate adenocarcinoma and overall survival in primary melanoma, lowgrade glioma, and kidney papillary cell carcinoma. ${ }^{29}$ WISP1 could regulate complex biological process during disease by effecting proliferation, cell survival, and cell differentiation, development and disease. ${ }^{10,16,30,31}$ It had been pointed out that WISP1 protects lung carcinoma cells from intrinsic p53-dependent, but not extrinsic Fas ligand-

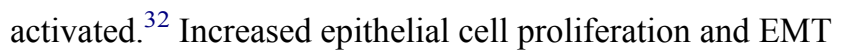
induction highlight WISP1 as a possible linker of organ fibrosis and cancer. ${ }^{33}$ The correlation of its expression with clinical outcome makes WISP1 a promising target for the evaluation of clinical diagnosis and prognosis of cancers. 


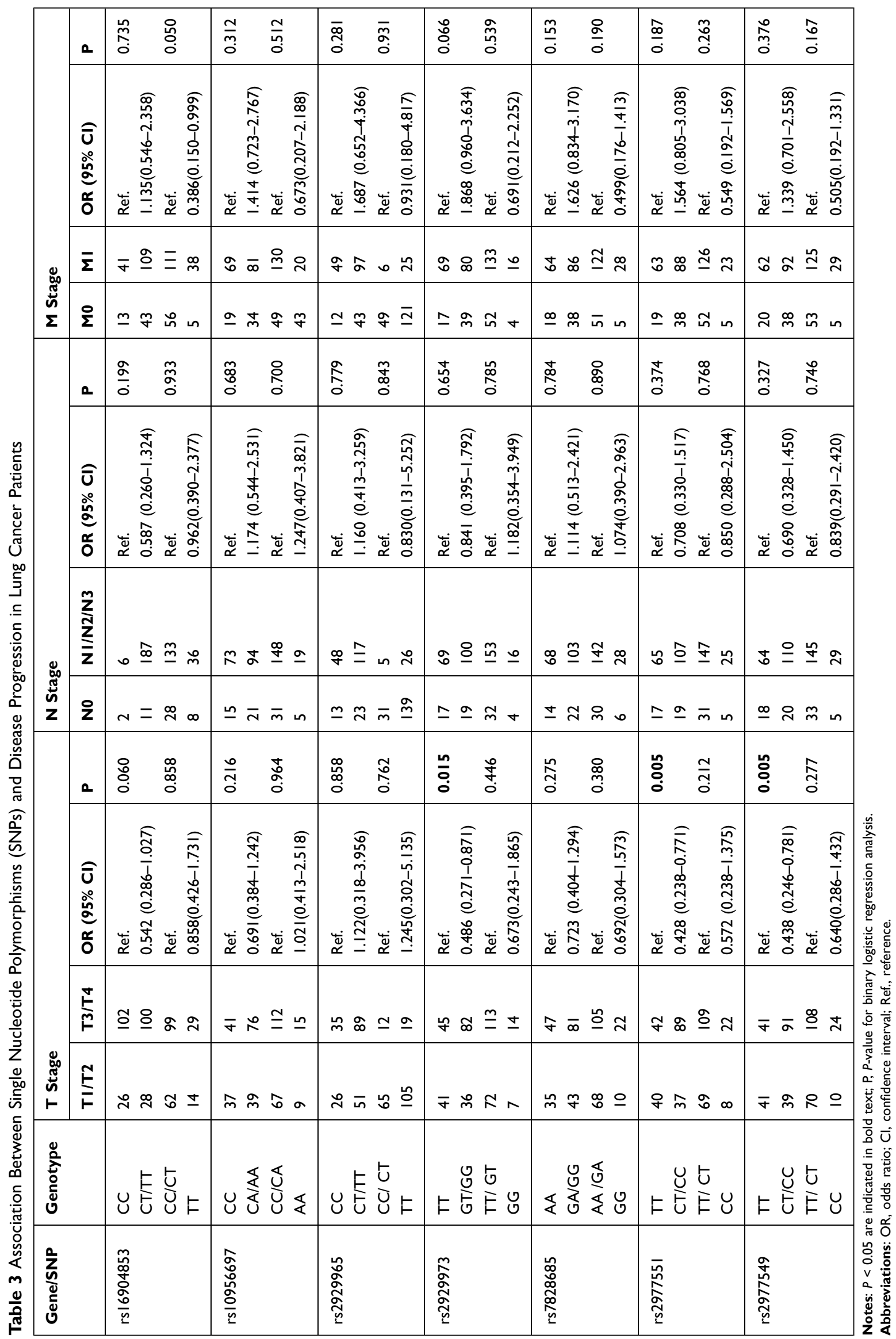


Table 4 Stratified Analysis of the Association Between rs2929973, rs297755I, rs2977549 and Prognosis in Lung Cancer Patients

\begin{tabular}{|c|c|c|c|c|c|c|c|c|c|}
\hline \multirow[t]{3}{*}{ SNP } & \multirow[t]{3}{*}{ Subgroups } & \multicolumn{4}{|c|}{ Overall Survival (OS) } & \multicolumn{4}{|c|}{ Progression-Free Survival (PFS) } \\
\hline & & \multicolumn{2}{|c|}{ Death/Total } & \multirow[t]{2}{*}{ HR $(95 \% \mathrm{Cl})$} & \multirow[t]{2}{*}{$\mathbf{P}$} & \multicolumn{2}{|c|}{ Progression/Total } & \multirow[t]{2}{*}{ HR (95\% Cl) } & \multirow[t]{2}{*}{$\mathbf{P}$} \\
\hline & & II & $12 / 22$ & & & II & $12 / 22$ & & \\
\hline \multirow[t]{4}{*}{ rs2929973 } & $\begin{array}{l}\text { Age (years) } \\
\leq 60\end{array}$ & $45 / 62$ & $109 / 120$ & $0.650(0.458-0.924)$ & 0.016 & $55 / 90$ & $120 / 148$ & $1.535(1.114-2.116)$ & 0.009 \\
\hline & $\begin{array}{l}\text { Smoking status } \\
\text { Never smoker }\end{array}$ & $24 / 33$ & $64 / 71$ & $0.617(0.383-0.994)$ & 0.047 & $31 / 50$ & $69 / 85$ & $1.457(0.952-2.231)$ & 0.083 \\
\hline & $\begin{array}{r}\text { Histology } \\
\text { NSCLC }\end{array}$ & $57 / 70$ & $109 / 117$ & $0.693(0.50 I-0.959)$ & 0.027 & $67 / 97$ & $117 / 143$ & $1.338(0.990-1.808)$ & 0.058 \\
\hline & $\begin{array}{l}\text { TNM stage } \\
\text { III/IV } \\
\text { ED }\end{array}$ & $\begin{array}{l}59 / 75 \\
10 / 10\end{array}$ & $\begin{array}{l}107 / 114 \\
25 / 27\end{array}$ & $\begin{array}{l}0.649(0.470-0.896) \\
1.002(0.466-2.156)\end{array}$ & $\begin{array}{l}\mathbf{0 . 0 0 9} \\
0.995\end{array}$ & $\begin{array}{l}70 / 102 \\
10 / 19\end{array}$ & $\begin{array}{l}119 / 143 \\
28 / 32\end{array}$ & $\begin{array}{l}1.377(1.024-1.852) \\
2.550(1.188-5.473)\end{array}$ & $\begin{array}{l}0.034 \\
0.016\end{array}$ \\
\hline \multirow[t]{2}{*}{ rs297755। } & $\begin{array}{l}\text { Age (years) } \\
\leq 60\end{array}$ & $26 / 28$ & $130 / 154$ & $1.222(0.800-1.867)$ & 0.353 & $53 / 85$ & $12|/| 53$ & $1.413(1.022-1.954)$ & 0.037 \\
\hline & $\begin{array}{l}\text { Smoking status } \\
\text { Never smoker }\end{array}$ & $11 / 12$ & $36 / 47$ & $1.858(0.434-1.695)$ & 0.660 & $27 / 45$ & $71 / 89$ & $1.572(1.007-2.455)$ & 0.047 \\
\hline \multirow[t]{3}{*}{ rs2977549 } & $\begin{array}{l}\text { Age (years) } \\
\leq 60\end{array}$ & $54 / 66$ & $104 / 117$ & $1.178(0.846-1.639)$ & 0.332 & $54 / 84$ & $117 / 145$ & I.480(I.07I-2.047) & 0.018 \\
\hline & $\begin{array}{c}\text { Histology } \\
\text { NSCLC } \\
\text { SCLC }\end{array}$ & $\begin{array}{l}56 / 68 \\
82 / 100\end{array}$ & $\begin{array}{l}114 / 122 \\
165 / 182\end{array}$ & $\begin{array}{l}1.502(1.087-2.075) \\
1.288(0.987-1.682)\end{array}$ & $\begin{array}{l}0.014 \\
0.063\end{array}$ & $\begin{array}{l}66 / 93 \\
77 / 119\end{array}$ & $\begin{array}{l}51 / 121 \\
133 / 176\end{array}$ & $\begin{array}{l}1.358(1.005-1.835) \\
1.350(1.018-1.791)\end{array}$ & $\begin{array}{l}0.046 \\
0.037\end{array}$ \\
\hline & $\begin{array}{l}\text { TNM stage } \\
\text { III/IV } \\
\text { ED }\end{array}$ & $\begin{array}{l}55 / 69 \\
11 / 11\end{array}$ & $\begin{array}{l}115 / 123 \\
24 / 26\end{array}$ & $\begin{array}{l}1.601(1.157-2.216) \\
0.765(0.362-1.615)\end{array}$ & $\begin{array}{l}\mathbf{0 . 0 0 5} \\
0.482\end{array}$ & $\begin{array}{l}66 / 95 \\
11 / 20\end{array}$ & $\begin{array}{l}126 / 155 \\
27 / 32\end{array}$ & $\begin{array}{l}1.419(1.053-1.913) \\
0.48 \mid(0.232-1.000)\end{array}$ & $\begin{array}{l}\mathbf{0 . 0 2 2} \\
0.050\end{array}$ \\
\hline
\end{tabular}

Notes: $P<0.05$ are indicated in bold text; $P, P$-value for multivariate Cox hazards regression analysis with adjustment for age, gender, and histology; $I I / I 2 / 22=$ wild type/ heterozygote/homozygote.

Abbreviations: HR, hazard ratio; Cl, confidence interval; Ref., reference; LUAD, lung adenocarcinoma; LUSC, lung squamous cell carcinoma.

WISP1 signaling is critically involved in cell survival and growth, through regulating PI3K and Akt. ${ }^{12}$ WISP1 led to Akt phosphorylation in epithelial and mesenchymal cells. ${ }^{15,34}$ In our study, we found that WISP1 was related to platinum-based chemotherapy response of lung cancer.

Previous association studies that polymorphism is one of the factors that may affect prognosis, mainly focused on the mis-sense variants or coding regions of individual genes. In this study, we focused on the genetic alteration of WISP1, a total of 39 SNPs to study the prognosis effect platinum-based chemotherapy. Our study found WISP1 rs2929973 carrying G variant allele are more likely to have an increased susceptibility of lung cancer. Patients with younger age $(\leq 60)$, or patients who had never smoked, NSCLC, or advanced tumor (TNM stage III/IV), it had better OS when rs2929973 carrying TT genotype. Patients who carry the rs2977551 CT or CC genotype tended to have a significantly increased risk of PFS in subgroups of younger ( $\leq 60$ years old), never smokers. Patients carrying TT genotype in rs 2977549 better OS in lung cancer patient with NSCLC, had TNM stage III/IV. In conclusion, the WISP1 rs2929973 T allele, rs2977551 $\mathrm{T}$ allele and rs2977549 $\mathrm{T}$ allele maybe a potential protective allele in the prognosis of lung cancer patients treated with platinum-based chemotherapy.

WISP1 is a downstream gene of the canonical Wnt- $\beta$ catenin pathway, and its mutations were reported to be associated with multiple diseases, including asthma, hypertension and spinal osteoarthritis. ${ }^{13,34-36}$ Chen et al revealed that WISP1 was overexpressed in non-small cell lung carcinoma (NSCLC) samples compared with their normal lung tissue counterparts. Our previous studies revealed that WISP1 genetic polymorphisms were related to susceptibility and the platinum-based chemotherapy response of lung cancer, and 
we hypothesized that WISP1 polymorphisms may also be associated with the chemotherapy toxicity of lung cancer. ${ }^{28}$ Most previous studies about the relationships of WISP1 polymorphisms and diseases were focused on the polymorphisms of WISP1 rs2929973. However, results from different studies often provide contradicting results or statistically nonsignificant associations. Previous study indicated that WISP1 rs2929973 was associated with asthma and individuals carrying the $\mathrm{G}$ allele of rs2929973 conferring lower forced expiratory volume in the first second. ${ }^{37}$ Patients carrying the WISP1 rs2929973 GG and TT variant were almost twice as likely as those carrying the GT genotype to have estrogen receptor (ER)- and progesterone receptor (PR)-positive tumors. ${ }^{38}$ In addition, the genetic polymorphisms of WISP1 (rs2977549) variants were associated with lung and smoking-related cancer prognosis. ${ }^{27}$ Previous research found that patient carrying the rs10956697 AC genotype had a significantly decreased risk of gastric cancer $(\mathrm{OR}=0.58,95 \% \mathrm{CI} 0.35-0.98)$. Smokers with the rs $10956697 \mathrm{AC}$ and AC + AA genotypes exhibited a 0.28fold lower and 0.32-fold lower risk of gastric cancer. ${ }^{39}$ Rs16904853, rs2977549, and rs2977551 polymorphisms were significantly associated with hematologic toxicity. ${ }^{27}$

However, our study has several limitations. Independent validation of correlations between association of SNPs with lung cancer prognosis are needed. No SNPs remained significant by considering multiple-testing correction that calculated the $\mathrm{p}$ value of the SNPs by FDR-BH (Benjamini \& Hochberg (1995) step-up FDR control) correction, it perhaps the sample size for the study is not large enough. Moreover, the function represent interconnections between polymorphisms and prognosis of lung cancer patients with platinumbased chemotherapy was not determined in our study.

\section{Conclusion}

In conclusion, lung cancer patients with WISP1 rs2929973 GT or GG, rs2977551 CT or CC and rs2977549 CT or CC genotypes had increased risk of disease progression, and patients with rs2977551 CC or CT had a significantly longer OS. Our results showed that those polymorphisms were significantly associated with the chemotherapy prognosis of lung cancer patients. Thus, we thought that the genotypes of WISP1 may be contributed to a potential candidate biomarker for the prediction of prognosis in lung cancer patients.

\section{Acknowledgments}

This work was supported by the National Nature Science Foundation of China (81803640), Hunan Provincial Natural Science Foundation of China (2020JJ5885).

\section{Disclosure}

The authors declare that they have no conflicts of interest.

\section{References}

1. McGuire S. World cancer report 2014. Geneva, Switzerland: World Health Organization, international agency for research on cancer, WHO Press, 2015. Adv Nutr. 2016;7(2):418-419. doi:10.3945/ an. 116.012211

2. Chen W, Zheng R, Zeng H, Zhang S. The updated incidences and mortalities of major cancers in China, 2011. Chin J Cancer. 2015;34 (11):502-507. doi:10.1186/s40880-015-0042-6

3. Li C, Yin M, Wang LE, et al. Polymorphisms of nucleotide excision repair genes predict melanoma survival. J Invest Dermatol. 2013;133 (7):1813-1821. doi:10.1038/jid.2012.498

4. Herbst RS, Heymach JV, Lippman SM. Lung cancer. N Engl J Med. 2008;359(13):1367-1380. doi:10.1056/NEJMra0802714

5. Hirsch FR, Scagliotti GV, Mulshine JL, et al. Lung cancer: current therapies and new targeted treatments. Lancet. 2017;389 (10066):299-311. doi:10.1016/S0140-6736(16)30958-8

6. Way K. Working against racism. Interview by Lyndon Keene. N Z Nurs J. 1988;81(8):17-19.

7. Sandler A, Gray R, Perry MC, et al. Paclitaxel-carboplatin alone or with bevacizumab for non-small-cell lung cancer. $N$ Engl $J$ Med. 2006;355(24):2542-2550. doi:10.1056/NEJMoa061884

8. Scagliotti GV, Parikh P, von Pawel J, et al. Phase III study comparing cisplatin plus gemcitabine with cisplatin plus pemetrexed in chemotherapy-naive patients with advanced-stage non-small-cell lung cancer. J Clin Oncol. 2008;26(21):3543-3551. doi:10.1200/ JCO.2007.15.0375

9. Berschneider B, Konigshoff M. WNT1 inducible signaling pathway protein 1 (WISP1): a novel mediator linking development and disease. Int J Biochem Cell Biol. 2011;43(3):306-309. doi:10.1016/ j.biocel.2010.11.013

10. Pennica D, Swanson TA, Welsh JW, et al. WISP genes are members of the connective tissue growth factor family that are up-regulated in wnt-1-transformed cells and aberrantly expressed in human colon tumors. Proc Natl Acad Sci U S A. 1998;95(25):14717-14722. doi:10.1073/pnas.95.25.14717

11. Jun JI, Lau LF. Taking aim at the extracellular matrix: CCN proteins as emerging therapeutic targets. Nat Rev Drug Discov. 2011;10 (12):945-963. doi:10.1038/nrd3599

12. Venkatesan B, Prabhu SD, Venkatachalam K, et al. WNT1-inducible signaling pathway protein-1 activates diverse cell survival pathways and blocks doxorubicin-induced cardiomyocyte death. Cell Signal. 2010;22(5):809-820. doi:10.1016/j.cellsig.2010.01.005

13. Venkatachalam K, Venkatesan B, Valente AJ, et al. WISP1, a pro-mitogenic, pro-survival factor, mediates tumor necrosis factor-alpha (TNF-alpha)-stimulated cardiac fibroblast proliferation but inhibits TNF-alpha-induced cardiomyocyte death. J Biol Chem. 2009;284(21):14414-14427. doi:10.1074/jbc.M809757200

14. Inkson CA, Ono M, Bi Y, Kuznetsov SA, Fisher LW, Young MF. The potential functional interaction of biglycan and WISP-1 in controlling differentiation and proliferation of osteogenic cells. Cells Tissues Organs. 2009;189(1-4):153-157. doi:10.1159/000151377

15. Colston JT, de la Rosa SD, Koehler M, et al. Wnt-induced secreted protein-1 is a prohypertrophic and profibrotic growth factor. $\mathrm{Am}$ $J$ Physiol Heart Circ Physiol. 2007;293(3):H1839-1846. doi:10.1152/ajpheart.00428.2007

16. Calvisi DF, Conner EA, Ladu S, Lemmer ER, Factor VM, Thorgeirsson SS. Activation of the canonical Wnt/beta-catenin pathway confers growth advantages in c-Myc/E2F1 transgenic mouse model of liver cancer. J Hepatol. 2005;42(6):842-849. doi:10.1016/ j.jhep.2005.01.029 
17. Margalit O, Eisenbach L, Amariglio N, et al. Overexpression of a set of genes, including WISP-1, common to pulmonary metastases of both mouse D122 Lewis lung carcinoma and B16-F10.9 melanoma cell lines. $B r \quad J$ Cancer. 2003;89(2):314-319. doi:10.1038/sj. bjc. 6600977

18. Xie D, Nakachi K, Wang H, Elashoff R, Koeffler HP. Elevated levels of connective tissue growth factor, WISP-1, and CYR61 in primary breast cancers associated with more advanced features. Cancer Res. 2001;61(24):8917-8923

19. Seal A, Gupta A, Mahalaxmi M, Aykkal R, Singh TR, Arunachalam V. Tools, resources and databases for SNPs and indels in sequences: a review. Int $J$ Bioinform Res Appl. 2014;10 (3):264-296. doi:10.1504/IJBRA.2014.060762

20. Shastry BS. SNP alleles in human disease and evolution. J Hum Genet. 2002;47(11):561-566. doi:10.1007/s100380200086

21. Inkson CA, Ono M, Kuznetsov SA, Fisher LW, Robey PG, Young MF. TGF-beta1 and WISP-1/CCN-4 can regulate each other's activity to cooperatively control osteoblast function. J Cell Biochem. 2008;104(5):1865-1878. doi:10.1002/jcb.21754

22. Jun JI, Lau LF. The matricellular protein CCN1 induces fibroblast senescence and restricts fibrosis in cutaneous wound healing. Nat Cell Biol. 2010;12(7):676-685. doi:10.1038/ncb2070

23. Tian C, Zhou ZG, Meng WJ, et al. Overexpression of connective tissue growth factor WISP-1 in Chinese primary rectal cancer patients. World J Gastroenterol. 2007;13(28):3878-3882. doi:10.3748/wjg.v13.i28.3878

24. Nagai Y, Watanabe M, Ishikawa $S$, et al. Clinical significance of Wnt-induced secreted protein-1 (WISP-1/CCN4) in esophageal squamous cell carcinoma. Anticancer Res. 2011;31(3):991-997.

25. Wu J, Long Z, Cai H, et al. High expression of WISP1 in colon cancer is associated with apoptosis, invasion and poor prognosis. Oncotarget. 2016;7(31):49834-49847. doi:10.18632/oncotar get. 10486

26. Shao H, Cai L, Grichnik JM, Livingstone AS, Velazquez OC, Liu ZJ. Activation of Notch1 signaling in stromal fibroblasts inhibits melanoma growth by upregulating WISP-1. Oncogene. 2011;30 (42):4316-4326. doi:10.1038/onc.2011.142

27. Chen J, Yin J, Li X, et al. WISP1 polymorphisms contribute to platinum-based chemotherapy toxicity in lung cancer patients Int $J$ Mol Sci. 2014;15(11):21011-21027. doi:10.3390/ijms151 121011

28. Chen J, Yin JY, Li XP, et al. Association of Wnt-inducible signaling pathway protein 1 genetic polymorphisms with lung cancer susceptibility and platinum-based chemotherapy response. Clin Lung Cancer. 2015;16(4):298. doi:10.1016/j.cllc.2014.12.008
29. Gaudreau PO, Clairefond S, Class CA, et al. WISP1 is associated to advanced disease, EMT and an inflamed tumor microenvironment in multiple solid tumors. Oncoimmunology. 2019;8(5):e1581545. doi:10.1080/2162402X.2019.1581545

30. Blom AB, Brockbank SM, van Lent PL, et al. Involvement of the Wnt signaling pathway in experimental and human osteoarthritis: prominent role of Wnt-induced signaling protein 1. Arthritis Rheum. 2009;60(2):501-512. doi:10.1002/art.24247

31. Xu L, Corcoran RB, Welsh JW, Pennica D, Levine AJ. WISP-1 is a Wnt-1- and beta-catenin-responsive oncogene. Genes Dev. 2000;14 (5):585-595.

32. Su F, Overholtzer M, Besser D, Levine AJ. WISP-1 attenuates p53-mediated apoptosis in response to DNA damage through activation of the Akt kinase. Genes Dev. 2002;16(1):46-57. doi:10.1101/ gad. 942902

33. You Z, Saims D, Chen S, et al. Wnt signaling promotes oncogenic transformation by inhibiting c-Myc-induced apoptosis. J Cell Biol. 2002;157(3):429-440. doi:10.1083/jcb.200201110

34. Konigshoff M, Kramer M, Balsara N, et al. WNT1-inducible signaling protein-1 mediates pulmonary fibrosis in mice and is upregulated in humans with idiopathic pulmonary fibrosis. J Clin Invest. 2009;119 (4):772-787.

35. Wang $H$, Zhang $\mathrm{R}$, Wen $\mathrm{S}$, McCafferty DM, Beck PL, MacNaughton WK. Nitric oxide increases Wnt-induced secreted protein-1 (WISP-1/CCN4) expression and function in colitis. $J$ Mol Med. 2009;87(4):435-445. doi:10.1007/s00109-009-0445-4

36. Witte F, Dokas J, Neuendorf F, Mundlos S, Stricker S. Comprehensive expression analysis of all Wnt genes and their major secreted antagonists during mouse limb development and cartilage differentiation. Gene Expr Patterns. 2009;9(4):215-223. doi:10.1016/j.gep.2008.12.009

37. Sharma S, Tantisira K, Carey V, et al. A role for Wnt signaling genes in the pathogenesis of impaired lung function in asthma. Am J Respir Crit Care Med. 2010;181(4):328-336. doi:10.1164/rccm.200907$10090 \mathrm{OC}$

38. Lee HL, Chiou HL, Wang SS, et al. WISP1 genetic variants as predictors of tumor development with urothelial cell carcinoma. Urol Oncol. 2018;36(4):160e115-160e121. doi:10.1016/j. urolonc.2017.11.023

39. Liu Y, Qin W, Zhang F, et al. Association between WNT-1-inducible signaling pathway protein-1 (WISP1) genetic polymorphisms and the risk of gastric cancer in Guangxi Chinese. Cancer Cell Int. 2021;21 (1):405. doi:10.1186/s12935-021-02116-2
Pharmacogenomics and Personalized Medicine

\section{Publish your work in this journal}

Pharmacogenomics and Personalized Medicine is an international, peer-reviewed, open access journal characterizing the influence of genotype on pharmacology leading to the development of personalized treatment programs and individualized drug selection for improved safety, efficacy and sustainability. This journal is indexed on the American Chemical Society's Chemical Abstracts Service (CAS). The manuscript management system is completely online and includes a very quick and fair peer-review system, which is all easy to use. Visit http://www.dovepress.com/testimonials.php to read real quotes from published authors. 